\title{
MicroRNA-154 functions as a tumor suppressor in non-small cell lung cancer through directly targeting B-cell-specific Moloney murine leukemia virus insertion site 1
}

\author{
SIDA LIU ${ }^{1}$, YANG YANG ${ }^{2}, \mathrm{LU} \mathrm{CHEN}^{2}$, DANWEI LIU $^{3}$ and $\mathrm{HAN} \mathrm{DONG}^{4}$ \\ ${ }^{1}$ Department of Thoracic Surgery, XinHua Hospital Affiliated to Shanghai Jiao Tong University School of Medicine, \\ Shanghai 200092; ${ }^{2}$ Department of Tumor and Blood Disease, The Affiliated Hospital to Changchun University \\ of Chinese Medicine; ${ }^{3}$ Department of Infectious, People's Hospital of Jilin; ${ }^{4}$ Department of Geratology, \\ The First Hospital of Jilin University, Changchun, Jilin 130021, P.R. China
}

Received March 9, 2016; Accepted March 30, 2017

DOI: $10.3892 /$ ol.2018.8595

\begin{abstract}
Lung cancer remains the leading cause of cancer-associated mortality in China and worldwide. Increasing numbers of studies have demonstrated that microRNAs (miRNAs/miRs) have vital functions in numerous developmental processes and tumorigenesis. The aim of the present study was to investigate miR-154 expression in non-small cell lung cancer (NSCLC), and to explore the roles of miR-154 in the carcinogenesis and progression of this cancer. Reverse transcription-polymerase chain reaction (RT-qPCR) was performed to detect miR-154 expression in NSCLC tissues and cell lines. In addition, cell proliferation assay, migration and invasion assays were adopted to investigate the functional roles of miR-154 in NSCLC. Bioinformatics analysis, luciferase reporter assay, RT-qPCR and western blot analysis were used to explore the potential targets of miR-154 in NSCLC. According to the results, miR-154 was significantly downregulated in NSCLC tissues and cell lines. Restoration of miR-154 expression inhibited proliferation, migration and invasion of NSCLC cells. In addition, B-cell-specific Moloney murine leukemia virus insertion site 1 (BMI-1) was identified as a direct target gene of miR-154 in NSCLC. In conclusion, miR-154 may function as a tumor suppressor in NSCLC, partly by regulating BMI-1, and the modulation of miR-154 expression represents a potential strategy for the treatment of NSCLC patients.
\end{abstract}

Correspondence to: Professor Han Dong, Department of Geratology, The First Hospital of Jilin University, 71 XinMin Street, Chaoyang, Changchun, Jilin 130021, P.R. China

E-mail: donghan6217111@163.com

Key words: non-small cell lung cancer, microRNA-154, B-cell-specific Moloney murine leukemia virus insertion site 1, proliferation, metastasis

\section{Introduction}

Lung cancer, a highly malignant tumor, is the leading cause of cancer-associated mortality in males and females worldwide (1). The incidence and mortality rates of non-small cell lung cancer are increasing in developing countries, including China, due to the increase in the use of tobacco as well as air pollution (2). According to its pathological pattern, lung cancer may be divided into small cell lung cancer (SCLC) and non-small cell lung cancer (NSCLC) (3). NSCLC, which includes adenocarcinoma, squamous cell carcinoma, adenosquamous cell carcinoma and large cell carcinoma, accounts for $\sim 80-85 \%$ of all lung cancer cases (4). Despite advances in traditional treatments, including surgery, supplemented with radiotherapy and chemotherapy, the prognosis remains poor and the five-year overall survival rate is extremely low (17.1\%) (5). These facts indicate an urgent requirement to fully understand the molecular mechanisms underlying the carcinogenesis and progression of NSCLC, and to investigate novel therapeutic targets to control this malignant disease.

Increasing studies have demonstrated that microRNAs (miRNAs/miRs) have vital functions in numerous developmental processes and tumorigenesis, including the progression of NSCLC (6-8). miRNAs represent a group of small ( 19-25 nucleotides), non-protein-coding, and endogenous single RNAs, which negatively regulate gene expression by binding to the $3^{\prime}$ untranslated regions (3'UTRs) of target genes in an imperfect base pairing manner, causing mRNA cleavage or translational repression $(9,10)$. To date, miRNAs have been demonstrated to regulate $>30 \%$ of all cellular proteins and serve substantial roles in a wide range of physiological and pathological processes, including cell growth, cell cycle, apoptosis, development, migration, invasion, survival and metastasis (11-13). Accumulating evidence suggests that the abnormal expression of miRNAs is associated with various types of human cancer, and may serve as a new therapeutic strategy for cancers as they act as oncogenes or tumor suppressors in tumorigenesis and development (14).

In the present study, the potential roles of miR-154 were investigated in NSCLC. The expression levels of miR-154 
were measured in NSCLC tissues and cell lines, and its effects on cell proliferation, migration and invasion were evaluated. Furthermore, the direct targets of miR-154 in NSCLC and the underlining molecular mechanism of its functions were explored. The results are likely to provide a better understanding of NSCLC carcinogenesis and progression, and a therapeutic target for patients with NSCLC.

\section{Materials and methods}

Tissue samples, cell lines and cell transfection. This study was approved by the ethics committee of XinHua Hospital Affiliated to Shanghai Jiao Tong University School of Medicine, China. Written informed consent was obtained from all patients prior to enrollment in the present study. NSCLC tissues and matched normal lung tissues were collected from 32 patients at XinHua Hospital Affiliated to Shanghai Jiao Tong University School of Medicine. None of these NSCLC patients had received chemotherapy or radiotherapy prior to the surgery. Following collection, all tissue samples were immediately snap-frozen in liquid nitrogen and stored at $-80^{\circ} \mathrm{C}$.

All cell lines were purchased from the American Type Culture Collection (ATCC; Manassas, VA, USA). The normal human bronchial epithelial cell line 16HBE and NSCLC cell lines SK-MES-1, H520, SPC-A1 and A549 were cultured in Dulbecco's modified Eagle's medium (DMEM; Gibco; Thermo Fisher Scientific, Inc., Waltham, MA, USA) supplemented with $10 \%$ fetal bovine serum (FBS; Gibco), $100 \mathrm{U} / \mathrm{ml}$ penicillin and $100 \mu \mathrm{g} / \mathrm{ml}$ streptomycin, and maintained at $37^{\circ} \mathrm{C}$ in a $5 \% \mathrm{CO}_{2}$ humidified atmosphere.

The miR-154 mimics and negative control(NC) were obtained from GenePharma (Shanghai, China). pcDNA3.1-BMI-1 or pcDNA3.1-Ctl were synthesized by Guangzhou RiboBio Co., Ltd. (Guangzhou, China). For transfection, cells were seeded in six-well plates and grown to a confluency of 50-60\%. Transfections were performed using a Lipofectamine 2000 kit (Invitrogen; Thermo Fisher Scientific, Inc., Waltham, MA, USA) following the manufacturer's protocol.

$R N A$ isolation and reverse transcription-quantitative PCR $(R T-q P C R)$. Total RNA was isolated from tissues or cell lines using TRIzol reagent (Invitrogen) according to the standard protocol. The concentration and purity of total RNA were determined by A260/A280 with a NanoDrop ND-2000 spectrophotometer (NanoDrop Technologies; Thermo Fisher Scientific, Inc., Wilmington, DE, USA). For miR-154 expression, total RNA was reversed transcribed into cDNA using a TaqMan microRNA reverse transcription kit (Applied Biosystems; Thermo Fisher Scientific, Inc., Waltham, MA, USA). qPCR was performed with a TaqMan microRNA assay kit (Applied Biosystems). For BMI-1 mRNA expression, the synthesis of cDNA was performed using an M-MLV First Strand kit (Invitrogen), followed by qPCR with a SYBR-Green PCR kit (Takara Biotechnology Co., Ltd., Dalian, China). U6 and GAPDH mRNA were used as endogenous controls for miR-154 and BMI-1 mRNA expression levels. All reactions were performed on an ABI 7500 real-time system (Applied Biosystems).

Cell proliferation assay. Cell proliferation was evaluated using a 3-(4,5-dimethylthiazol-2-yl)-2,5-diphenyltetrazolium bromide (MTT) assay, according to the manufacturer's protocol. In briefly, transfected cells were harvested, seeded in 96-well plates at a concentration of 3,000 cells per well, and cultured for 1, 2, 3 and 4 days. At the indicated time points, cells were incubated with $20 \mu \mathrm{l}$ MTT solution (5 mg/ml; Sigma-Aldrich; Merck KGaA, Darmstadt, Germany) for $4 \mathrm{~h}$ at $37^{\circ} \mathrm{C}$. Then, the MTT solution was removed and dimethyl sulfoxide was added into each well to dissolve the formazan crystals. Optical density was determined using a microplate reader (Pharmacia Biotech, Uppsala, Sweden) at a wavelength of $490 \mathrm{~nm}$.

Migration and invasion assay. For migration assays, transfected cells were collected, suspended with FBS-free culture medium, and seeded in the upper chambers of Transwell plates (BD Biosciences, San Jose, CA, USA). DMEM containing 20\% FBS was added to the lower chamber as a chemoattractant. The Transwell plates were incubated in $5 \% \mathrm{CO}_{2}$ at $37^{\circ} \mathrm{C}$ for $24 \mathrm{~h}$. The cells in the upper chamber were carefully removed with cotton swabs, then the plates were fixed in $95 \%$ methanol and stained with $0.1 \%$ crystal violet. The stained cells were counted in five random fields per Transwell plate under a microscope, and quantification was performed by manually counting the stained cells. The invasion assays were carried out in the same way as migration assays, with the exception that Matrigel (BD Biosciences) was used in the Transwell plates.

Bioinformatics methods. TargetScan (http://www.targetscan. org/) and PicTar (http://pictar.mdcberlin.de/) were used to predict the potential target genes of miR-154.

Luciferase reporter assay. The 3'UTR of BMI-1 containing a putative binding site (BMI-1-3'UTR WT) or a mutant (BMI-1-3'UTR MUT) cloned into the psi-CHECK2 vectors were synthesized by GenePharma. HEK293T cells were seeded in 24-well plates. Following incubation overnight, BMI-1-3'UTR WT and BMI-1-3'UTR MUT were co-transfected with miR-154 mimics or NC using Lipofectamine 2000 reagent. Forty-eight h after transfection, cells were collected, and firefly and renilla luciferase activity was detected using a dual luciferase reporter assay system (Promega Corporation, Madison, WI, USA) according to the manufacturer's protocol. Renilla luciferase was used as an internal control.

Western blot analysis. Proteins were isolated using radioimmunoprecipitation assay buffer (Sigma-Aldrich) containing $1 \mathrm{mM}$ phenylmethylsulfonyl fluoride (Sigma-Aldrich). Twenty micrograms of protein were loaded into $10 \%$ SDS-PAGE, and transferred to a polyvinylidene fluoride membrane (Bio-Rad Laboratories, Inc., Hercules, CA, USA). The membranes were then blocked for $30 \mathrm{~min}$ at room temperature with $5 \%$ skimmed milk in Tris-buffered saline (TBS) solution containing $0.1 \%$ Tween 20 (TBST), and probed with primary antibodies at $4^{\circ} \mathrm{C}$ overnight. The primary antibodies used in this study include mouse anti-human monoclonal BMI-1 antibody (sc-390443; 1:1,000; Santa Cruz Biotechnology, Inc., Dallas, TX, USA) and mouse anti-human monoclonal GADPH antibody (sc-365062; 1:1,000; Santa Cruz Biotechnology, Inc.). Subsequently, the membranes were washed with TBST three times and incubated with horseradish peroxidase-conjugated secondary antibody (1:5,000; Santa Cruz Biotechnology, Inc.) 

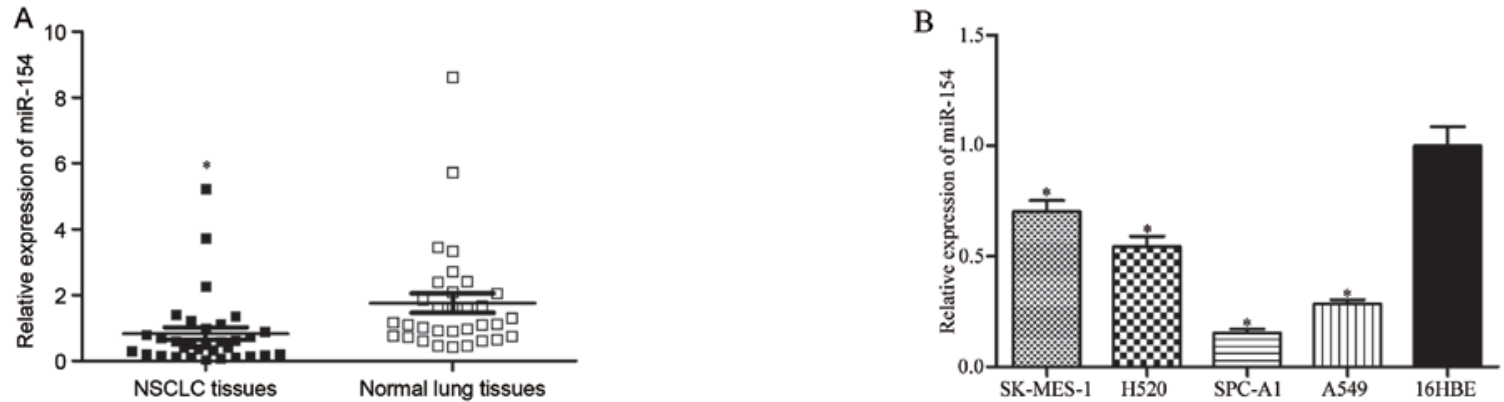

Figure 1. miR-154 is downregulated in NSCLC tissues and cell lines. (A) miR-154 expression levels in NSCLC tissues compared with matched normal lung tissues were determined using RT-qPCR. (B) miR-154 expression in four NSCLC cell lines and normal human bronchial epithelial cell line 16HBE was measured using RT-qPCR. "P<0.05, compared with normal group. miR, microRNA; NSCLC, non-small cell lung cancer; RT-qPCR, reverse transcription-quantitative polymerase chain reaction.
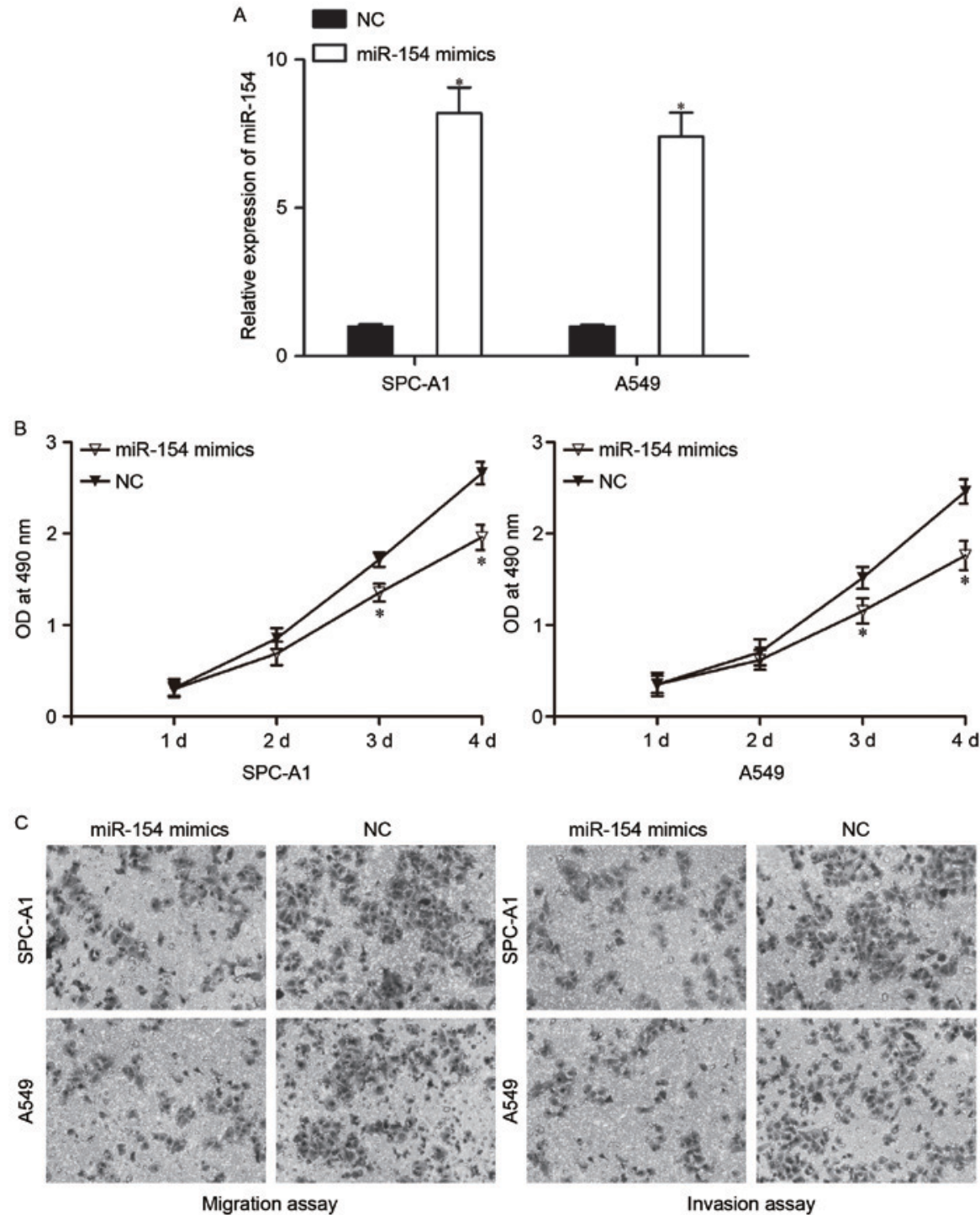

Figure 2. miR-154 inhibits NSCLC cell proliferation, migration and invasion. (A) RT-qPCR analysis was performed to detect miR-154 expression in SPC-A1 and A549 cells following transfection with miR-154 mimics or NC. (B) Cell proliferation assay was used to evaluate the effect of miR-154 overexpression on NSCLC cell proliferation. (C) Migration and invasion assays demonstrated that miR-154 overexpression decreased SPC-A1 and A549 cell migration and invasion capacity. Migrated and invaded cells were stained and fixed in $95 \%$ methanol, stained with $0.1 \%$ crystal violet, and images were captured (magnification, $\mathrm{x} 200$ ). ${ }^{*} \mathrm{P}<0.05$ compared with NC group. miR, microRNA; NSCLC, non-small cell lung cancer; RT-qPCR, reverse transcription-quantitative polymerase chain reaction; $\mathrm{NC}$, negative control; OD, optical density.

for $2 \mathrm{~h}$ at room temperature. Finally, the membranes were washed again with TBST three times and the proteins were visualized using an enhanced chemiluminescence detection system (GE Healthcare Life Sciences, Chalfont, UK). 
A
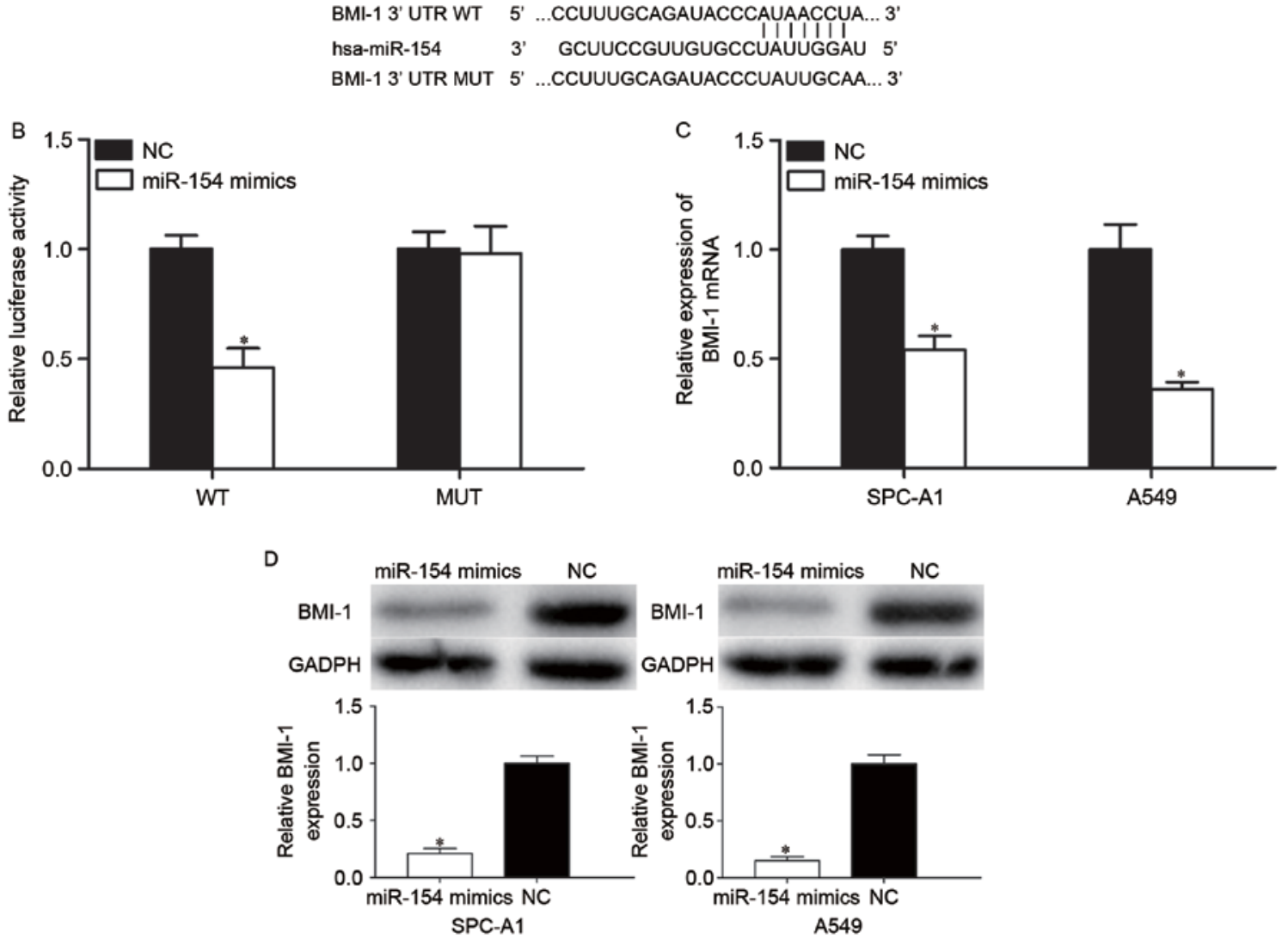

Figure 3. BMI-1 is a direct target gene of miR-154 in NSCLC. (A) Bioinformatics analysis revealed that the $3^{\prime} \mathrm{UTR}$ of BMI-1 contains a putative site that is partially complementary to miR-154. (B) Luciferase reporter assays revealed that miR-154 reduced the luciferase activity of BMI-1-3'UTR WT, while the activity of the HEK293T cells transfected with BMI-1-3'UTR MUT was not significantly different from that of the control group. (C) miR-154 overexpression decreased BMI-1 mRNA expression in SPC-A1 and A549 cells. (D) The expression of BMI-1 at the protein level in miR-154 mimic-transfected SPC-A1 and A549 cells was analyzed by western blot analysis. "P<0.05, compared with NC group. BMI-1, B-cell-specific Moloney murine leukemia virus insertion site 1 ; miR, microRNA; NSCLC, non-small cell lung cancer; 3'UTR, 3' untranslated region; WT, wild type; MUT, mutant; NC, negative control.

Statistical analysis. All data are presented as the means \pm standard deviation. SPSS 15.0 (SPSS Inc., Chicago, IL, USA) was used for statistical analyses. $\mathrm{P}<0.05$ was considered to indicate a statistically significant difference.

\section{Results}

miR-154 is downregulated in NSCLC. Firstly, RT-qPCR was performed to measure miR-154 expression in NSCLC tissues and matched normal lung tissues. The results revealed that miR-154 was significantly downregulated in NSCLC tissues in comparison with matched normal lung tissues (Fig. 1A, $\mathrm{P}<0.05)$. miR-154 expression levels in NSCLC cell lines and normal human bronchial epithelial cell line $16 \mathrm{HBE}$ were also determined using RT-qPCR. As shown in Fig. 1B, miR-154 expression levels were decreased in all four NSCLC cell lines compared with $16 \mathrm{HBE}(\mathrm{P}<0.05)$. Among the four NSCLC cell lines, SPC-A1 and A549 expressed the lowest miR-154 levels and were thus selected for further analyses.

Effects of miR-154 overexpression on NSCLC cell proliferation, migration and invasion. To evaluate the biological roles of miR-154 in NSCLC, the effects of miR-154 overexpression on NSCLC cell proliferation, migration and invasion were investigated. miR-154 mimics or NC were transfected into SPC-A1 and A549 cells. Following transfection for 48 h, RT-qPCR was carried out to assess the transfection efficiency. As shown in Fig. 2A, miR-154 was significantly elevated by miR-154 mimic transfection in SPC-A1 and A549 cells $(\mathrm{P}<0.05)$.

Cell proliferation assay results revealed that cell proliferation was notably reduced in SPC-A1 and A549 cells transfected with miR-154 mimics (Fig. $2 \mathrm{~B}, \mathrm{P}<0.05$ ). In addition, migration and invasion assays revealed that miR-154 decreased the migration and invasion of SPC-A1 and A549 cells compared with the NC groups (Fig. $2 \mathrm{C}, \mathrm{P}<0.05$ ). Taken together, these results indicate that miR-154 may act as a tumor suppressor in NSCLC progression.

BMI-1 is a direct target of miR-154 in NSCLC. To further reveal the molecular mechanisms underlying this tumor suppressor role of miR-154, TargetScan and PicTar were used to predict the potential target genes of miR-154. Bioinformatics analysis revealed that BMI-1 is a potential target of miR-154. Subsequently, luciferase reporter assays were adopted to check whether miR-154 directly targets the 3'UTR of BMI-1. Putative target sites of miR-154 in 3'-UTR of BMI-1 are presented in Fig. 3A. As shown in Fig. 3B, miR-154 decreased the luciferase activity of the BMI-1-3'UTR ( $<<0.05)$, whereas BMI-1-3'UTR MUT blocked this decrease $(\mathrm{P}>0.05)$. To further evaluate whether BMI-1 was modulated by miR-154, miR-154 mimic or 

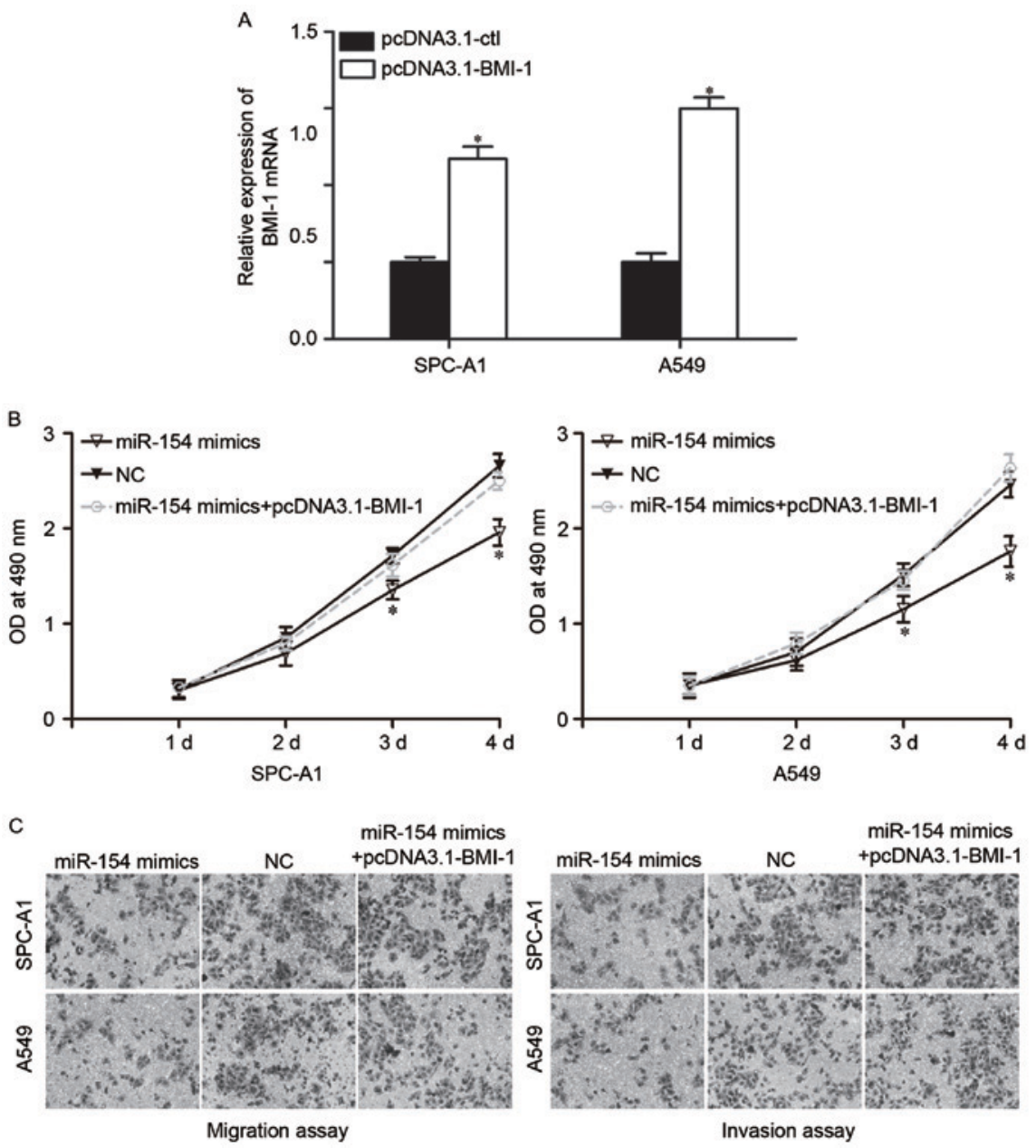

Figure 4. Overexpression of BMI-1 reverses the effects induced by miR-154 upregulation in NSCLC cells. (A) SPC-A1 and A549 cells were transfected with pcDNA3.1-BMI-1 or pcDNA3.1-Ct1. Forty-eight $\mathrm{h}$ after transfection, BMI-1 expression at the mRNA level was measured by RT-qPCR. "P<0.05, compared with pcDNA3.1-Ctl. Overexpression of BMI-1 reversed the inhibitory influence on the proliferation (B), migration and invasion (C) induced by miR-154 overexpression in SPC-A1 and A549 cells. Migrated and invaded cells were stained and fixed in 95\% methanol, stained with $0.1 \%$ crystal violet and imaged at magnification, $\mathrm{x} 200 .{ }^{*} \mathrm{P}<0.05$, compared with pcDNA3.1-Ctl. BMI-1, B-cell-specific Moloney murine leukemia virus insertion site 1; miR, microRNA; NSCLC, non-small cell lung cancer; RT-qPCR, reverse transcription-quantitative polymerase chain reaction; OD, optical density; NC, negative control.

NC was transfected into SPC-A1 and A549 cells, and BMI-1 expression levels were measured by RT-qPCR and western blot analysis. The results revealed that BMI-1 was significantly reduced at the mRNA (Fig. 3C, P<0.05) and protein (Fig. 3D, $\mathrm{P}<0.05)$ levels in miR-154-transfected SPC-A1 and A549 cells compared with the $\mathrm{NC}$ groups $(\mathrm{P}<0.05)$. These results suggested that miR-154 bound to the 3'UTR of BMI-1 and thus regulated its expression.

BMI-1 overexpression reverses effects of miR-154 upregulation in NSCLC cells. As BMI-1 was identified as a direct target of miR-154, it was then investigated whether BMI-1 had functional roles in regulating miR-154-induced NSCLC regulation. To do so, pcDNA3.1-BMI-1 or pcDNA3.1-Ctl were transfected into SPC-A1 and A549 cells. At $48 \mathrm{~h}$ after transfection, the expression levels of BMI-1 were determined by RT-qPCR (Fig. 4A, $\mathrm{P}<0.05$ ).

Next, various functional rescue experiments were performed. As expected, BMI-1 overexpression mostly reversed the inhibitory influence of miR-154 on proliferation (Fig. 4B, $\mathrm{P}<0.05$ ), migration and invasion (Fig. 4C, $\mathrm{P}<0.05$ ) in NSCLC cells. Thus, these data strongly demonstrate that
miR-154 acted as a tumor suppressor in NSCLC, at least in part through negative regulation of BMI-1.

\section{Discussion}

Lung cancer remains the leading cause of cancer-associated mortality in China and worldwide (15). With frequent local infiltration and distant metastasis, lung cancer development is a complex process that involves multiple genes, pathways and steps (16). A great deal of studies have indicated miRNAs to be critical regulators in the carcinogenesis and progression of human cancers (17-19). Furthermore, acting as either tumor suppressors or oncogenes, miRNAs have been demonstrated to be involved in a wide range of physiological and pathological processes, including cell growth, cell cycle, apoptosis, migration, invasion and metastasis (11-13). Therefore, exploring the correlation between NSCLC and miRNAs may be of benefit in the investigation of therapeutic strategies to improve the cure and survival rates of this cancer.

The data from the present study revealed that miR-154 was significantly downregulated in NSCLC tissues and cell lines, which was consistent with previous findings that expression 
levels of miR-154 were lower in several human cancer types and cell lines, including colorectal cancer (20), NSCLC (21), osteosarcoma (22), hepatocellular carcinoma (23) and prostate cancer (24). This finding indicated that the low expression levels of miR-154 may contribute to the carcinogenesis and progression of NSCLC. In addition, functional analysis revealed that restoration of miR-154 expression in NSCLC cells led to a significant inhibition of cell proliferation, migration and invasion. Next, BMI-1 was identified as a direct target gene of miR-154 in NSCLC via bioinformatics analysis, luciferase reporter assay, RT-qPCR and western blot analysis. In addition, BMI-1 overexpression reversed the inhibitory influence on NSCLC cells induced by miR-154, indicating that miR-154 acted as a tumor suppressor at least in part through the negative regulation of BMI-1.

The prognostic value of miR-154 has been investigated in several types of human cancer. For example, Kai et al reported that, in colorectal cancer, decreased expression levels of miR-154 were significantly associated with large tumor size, positive lymph node metastasis and advanced clinical stage (20). Univariate analysis revealed that colorectal cancer patients with low miR-154 expression levels had a poorer overall survival rate. In addition, multivariate analysis identified low miR-154 expression as an independent predictor of poor survival (20). Pang et al demonstrated that miR-154 expression was negatively correlated with tumor differentiation, tumor-node-metastasis (TNM) stage and lymph node metastasis in hepatocellular carcinoma (23). Lin et al revealed that low miR-154 expression was significantly correlated with metastasis, larger tumor size and advanced TNM stage in NSCLC (21). These findings implicated the potential effects of miR-154 in the prognosis of cancer.

The downregulation of miR-154 in several cancer types indicates that it may serve a significant role in the carcinogenesis and progression of cancer. Indeed, miR-154 has been demonstrated to be involved in several tumor suppressor functions. Zhou et al observed that miR-154 suppressed osteosarcoma cell proliferation, colony formation, migration and invasion, as well as inducing cell cycle arrest at the G1 stage (22). Pang et al reported that enforced miR-154 expression in hepatocellular carcinoma cells decreased cell growth and metastasis, and enhanced apoptosis and cell arrest at the G1 phase in vitro, as well as inhibiting tumor growth in vivo (23). Furthermore, miR-154 was noted to serve an essential role in regulating the growth, colony formation, migration and invasion of colorectal cancer cells (25). In prostate cancer, ectopic miR-154 expression inhibited proliferation, migration and invasion $(24,26)$. These findings indicated that miR-154 could be investigated as a therapeutic target for these human cancer types.

With regard to miR-154, several targets have been determined in previous studies, including Wnt5a in osteosarcoma (22), Zinc finger E-box-binding homeobox 2 in hepatocellular carcinoma (23), toll-like receptor 2 in colorectal cancer (25), and high-mobility group AT-hook 2 (24) and cyclin D2 (26) in prostate cancer. In the present study, a novel direct target gene of miR-154, BMI-1, was identified. Bioinformatics analysis revealed that BMI-1 was one of the potential target genes of miR-154. Luciferase reporter assays revealed that luciferase activity was suppressed by cotransfecting miR-154 mimics and BMI-1-3'UTR WT. However, this inhibition could be abrogated by cotransfecting miR-154 mimic and BMI-1-3'UTR MUT. Restoration of miR-154 expression decreased the expression of BMI-1 at the mRNA and protein expression level in NSCLC cells. Finally, BMI-1 overexpression reversed the inhibitory influence on NSCLC cells induced by miR-154. These findings indicated that targeting BMI-1 was involved in the tumor suppressor functions of miR-154 in NSCLC.

In conclusion, the present study offers evidence that miR-154 is downregulated in NSCLC and may act as a tumor suppressor in NSCLC carcinogenesis and progression, partly by negatively regulating BMI-1. Modulating miR-154 expression represents a potential strategy for the treatment of NSCLC patients.

\section{References}

1. Molina JR, Yang P, Cassivi SD, Schild SE and Adjei AA: Non-small cell lung cancer: Epidemiology, risk factors, treatment, and survivorship. Mayo Clin Proc 83: 584-594, 2008.

2. Yang L, Parkin DM, Li L and Chen Y: Time trends in cancer mortality in China: 1987-1999. Int J Cancer 106: 771-783, 2003.

3. Spira A and Ettinger DS: Multidisciplinary management of lung cancer. N Engl J Med 350: 379-392, 2004.

4. Goldstraw P, Ball D, Jett JR, Le Chevalier T, Lim E, Nicholson AG and Shepherd FA: Non-small-cell lung cancer. Lancet 378: 1727-1740, 2011.

5. Sánchez de Cos J, Sojo González MA, Montero MV, Pérez Calvo MC, Vicente MJ and Valle MH: Non-small cell lung cancer and silent brain metastasis. Survival and prognostic factors. Lung Cancer 63: 140-145, 2009.

6. Chen T, Xu C, Chen J, Ding C, Xu Z, Li C and Zhao J: MicroRNA-203 inhibits cellular proliferation and invasion by targeting Bmil in non-small cell lung cancer. Oncol Lett 9: 2639-2646, 2015.

7. Hou Y, Zhen J, Xu X, Zhen K, Zhu B, Pan R and Zhao C: miR-215 functions as a tumor suppressor and directly targets ZEB2 in human non-small cell lung cancer. Oncol Lett 10: 1985-1992, 2015.

8. Li D, Wei Y, Wang D, Gao H and Liu K: MicroRNA-26b suppresses the metastasis of non-small cell lung cancer by targeting MIEN1 via NF-kappaB/MMP-9/VEGF pathways. Biochem Biophys Res Commun 472: 465-470, 2016.

9. Bartel DP: MicroRNAs: Genomics, biogenesis, mechanism, and function. Cell 116: 281-297, 2004.

10. Engels BM and Hutvagner G: Principles and effects of microRNA-mediated post-transcriptional gene regulation. Oncogene 25: 6163-6169, 2006.

11. Aigner A: MicroRNAs (miRNAs) in cancer invasion and metastasis: Therapeutic approaches based on metastasis-related miRNAs. J Mol Med (Berl) 89: 445-457, 2011.

12. Rottiers V and Näär AM: MicroRNAs in metabolism and metabolic disorders. Nat Rev Mol Cell Biol 13: 239-250, 2012.

13. Cho WC: MicroRNAs: Potential biomarkers for cancer diagnosis, prognosis and targets for therapy. Int J Biochem Cell Biol 42: 1273-1281, 2010.

14. Li J, Wang Y, Luo J, Fu Z, Ying J, Yu Y and Yu W: miR-134 inhibits epithelial to mesenchymal transition by targeting FOXM1 in non-small cell lung cancer cells. FEBS Lett 586: 3761-3765, 2012.

15. Siegel R, Naishadham D and Jemal A: Cancer statistics, 2013. CA Cancer J Clin 63: 11-30, 2013.

16. Celli BR: Chronic obstructive pulmonary disease and lung cancer: Common pathogenesis, shared clinical challenges. Proc Am Thorac Soc 9: 74-79, 2012.

17. Jiang YW and Chen LA: microRNAs as tumor inhibitors, oncogenes, biomarkers for drug efficacy and outcome predictors in lung cancer (review). Mol Med Rep 5: 890-894, 2012.

18. Volinia S, Calin GA, Liu CG, Ambs S, Cimmino A, Petrocca F, Visone R, Iorio M, Roldo C, Ferracin M, et al: A microRNA expression signature of human solid tumors defines cancer gene targets. Proc Natl Acad Sci USA 103: 2257-2261, 2006.

19. Mishra PJ and Merlino G: MicroRNA reexpression as differentiation therapy in cancer. J Clin Invest 119: 2119-2123, 2009. 
20. Kai Y, Qiang C, Xinxin P, Miaomiao Z and Kuailu L: Decreased miR-154 expression and its clinical significance in human colorectal cancer. World J Surg Oncol 13: 195, 2015.

21. Lin X, Yang Z, Zhang P and Shao G: miR-154 suppresses non-small cell lung cancer growth in vitro and in vivo. Oncol Rep 33: 3053-3060, 2015.

22. Zhou H, Zhang M, Yuan H, Zheng W, Meng C and Zhao D MicroRNA-154 functions as a tumor suppressor in osteosarcoma by targeting Wnt5a. Oncol Rep 35: 1851-1858, 2016.

23. Pang X, Huang $K$, Zhang Q, Zhang Y and Niu J: miR-154 targeting ZEB2 in hepatocellular carcinoma functions as a potential tumor suppressor. Oncol Rep 34: 3272-3279, 2015.
24. Zhu C, Li J, Cheng G, Zhou H, Tao L, Cai H, Li P, Cao Q, Ju X, Meng X, et al: miR-154 inhibits EMT by targeting HMGA2 in prostate cancer cells. Mol Cell Biochem 379: 69-75, 2013.

25. Xin C, Zhang H and Liu Z: miR-154 suppresses colorectal cancer cell growth and motility by targeting TLR2. Mol Cell Biochem 387: 271-277, 2014

26. Zhu C, Shao P, Bao M, Li P, Zhou H, Cai H, Cao Q, Tao L, Meng X, Ju X, et al: miR-154 inhibits prostate cancer cell proliferation by targeting CCND2. Urol Oncol 32: 31.e9-e16, 2014. 